\title{
УДК 621.039.623 \\ COMPUTATIONS OF TOROIDAL ALFVÉN MODES IN SPHERICAL TOKAMAK GLOBUS-M PLASMAS
}

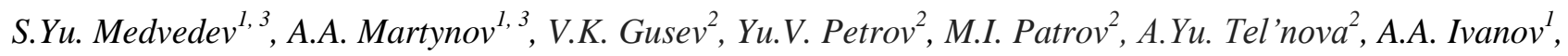 \\ Yu.Yu. Poshekhonov ${ }^{1}$
}

${ }^{I}$ Keldysh Institute of Applied Mathematics RAS, Moscow, Russia

${ }^{2}$ Ioffe Physical Technical Institute RAS, St. Petersburg, Russia

${ }^{3}$ NRC «Kurchatov Institute», Moscow, Russia

To determine the conditions for the excitation of TAE (Toroidal Alfvén Eigenmodes) and their influence on the confinement of fast particles in the spherical Globus-M tokamak [1-4], the MHD spectra were computed for the reconstructed divertor equilibrium configurations with the value of the safety factor at the magnetic axis $q_{0}>1$. The equilibrium reconstruction procedure provides the plasma shape and profiles including the electron temperature and density distributions along the normalized toroidal magnetic flux. The sensitivity of TAE frequencies with a toroidal wave number $n=1$ to the type of boundary conditions and the choice of the plasma boundary was investigated. It is found that the frequencies of the modes with dominant poloidal harmonics $m=1$ and $m=2$ in the continuum gap are much higher than those observed in the spectrograms of the signals from Mirnov coils, especially under the assumption of a free plasma boundary and taking into account plasma compressibility. The modes with lower frequencies and with higher poloidal wave numbers localized near the plasma boundary can be responsible for the oscillations observed in the experiment. However, for these modes a coupling to the continuum and possibly associated damping are expected.

Key words: tokamak, plasma, MHD spectrum, toroidal Alfvén eigenmodes.

\section{РАСЧЁТЫ ТОРОИДАЛЬНЫХ АЛЬФВЕНОВСКИХ МОД В ПЛАЗМЕ СФЕРИЧЕСКОГО ТОКАМАКА ГЛОБУС-М}

\author{
С.Ю. Медведев ${ }^{1,3}$, А.А. Мартынов ${ }^{1,3}$, В.К. Гусев ${ }^{2}$ Ю.В. Петров ${ }^{2}$, М.И. Патров ${ }^{2}$, А.Ю. Тельнова \\ А.А. Иванов ${ }^{1}$ Ю.Ю. Почехонов
}

\begin{abstract}
${ }^{1}$ Институт прикладной математики им. М.В. Келдыша РАН, Москва, Россия
${ }^{2}$ Физико-технический институт им. А.Ф. Иоффе РАН, Санкт-Петербург, Россия

${ }^{3}$ НИЦ «Курчатовский институт», Москва, Россия
\end{abstract}

Для выяснения условий возбуждения тороидальных альфвеновских собственных мод (ТАСМ) и их влияния на удержание быстрых частиц в сферическом токамаке Глобус-М проведены расчёты магнитогидродинамических спектров реконструированных диверторных равновесных конфигураций с значением фактора запаса устойчивости на магнитной оси $q_{0}>1$ [1-4]. Реконструкция равновесных конфигураций позволяет определить форму и профили характеристик плазмы, в частности, распределение электронной температуры и плотности вдоль нормализованного тороидального магнитного потока. Исследована чувствительность частот ТАСМ с тороидальным волновым числом $n=1$ по отношению к типу граничных условий и выбору граничной поверхности плазмы. Выяснено, что частоты мод с доминирующими полоидальными гармониками $m=1$ и $m=2$ в щели континуума существенно выше, чем наблюдаемые в спектрограммах сигналов от катушек Мирнова, особенно в предположении свободной границы плазмы и с учётом сжимаемости плазмы. ТАСМ с более низкими частотами и более высокими полоидальными волновыми числами, локализованные вблизи границы плазмы, могут быть ответственны за колебания, наблюдаемые в экспериментах. Однако для этих мод характерно взаимодействие с континуумом и, возможно, связанное с этим их затухание.

Ключевые слова: токамак, плазма, МГД-спектр, тороидальные альфвеновские собственные моды.

DOI: 10.21517/0202-3822-2018-41-2-95-104

\section{INTRODUCTION}

The practical use of spherical tokamaks is associated with the realization of projects involving compact neutron sources (CNS) that are currently being developed. Such projects assume implementation of neutral beam injection (NBI) into plasma with non-thermonuclear parameters. The main neutron rate is expected due to the interaction of fast ions produced, as a result of charge exchange of the beam atoms with thermal ions of the target plasma. The existence of fast particles with velocities exceeding the Alfvén speed in the plasma will lead to excitation of Alfvén instabilities provoking additional fast particle radial transport and losses thus decreasing the neutron rate. As shown in experiments on the tokamaks TFTR and D-IIID the toroidal Alfvén eigenmodes (TAE) [5] are the most dangerous as concerns fast particle redistribution and losses. In the mentioned experiments it was impossible to distinguish the radial redistribution of the fast ions from their total losses. During the past decade, the diagnostics have appeared that can provide direct measurements of expelled ions caused by TAE. In spherical tokamaks (as they operate at rather low toroidal fields), the fast ion velocity can be many 
times the Alfvén one, favoring the excitation of a wide range of instabilities. Experiments on the spherical tokamaks START, MAST and NSTX have indeed demonstrated excitation of different instabilities in the Alfvén frequency range including TAE. The parameters of these tokamaks permitted the development of several modes simultaneously with different toroidal numbers. At high enough amplitudes, the modes started to interplay with each other, resulting in the formation of so-called Alfvén avalanches [6]. As was shown in the experiments, they lead exactly to the largest fast particle redistribution and losses. So on NSTX, short TAE bursts of $1 \mathrm{~ms}$ duration resulted in the drop of the neutron rate by $40 \%$ associated with losses and redistribution of the fast particles. Thus, the modes in the Alfvén frequency band interact with magnetohydrodynamic (MHD) modes of lower frequency, such as fishbone and kink modes. As a result, it is very hard to understand which mode contributes to the losses. In the spherical tokamak Globus-M due to the smaller size as compared to the NSTX and MAST, TAE excitation is observed with strongly developed single modes $[1,2]$ in the sense that they correspond to the unique toroidal wave number, mostly $n=1$. That allows us to identify the effect of a single TAE on the fast particle confinement. As for a neutron source the main target parameter is the neutron rate, the results of experiments on Globus-M to study the effect of TAE on fast particle confinement deserve further theoretical and experimental investigations. The structure of the MHD spectrum and the spatial localization of the TAE in incompressible and compressible plasmas under different boundary conditions is the subject of the present paper. The purpose is to identify experimentally observed modes by their frequency and localization leaving the modeling of the mode destabilization by interaction with fast particles as well as the corresponding fast particle losses for a future work.

\section{PREPARATION OF EXPERIMENTAL DATA}

In the course of experiments on observation of TAE at the tokamak Globus-M Thomson scattering diagnostics have been used to measure the profiles of electron temperature and concentration. Based on these measurements the data for calculations of MHD spectra were prepared with the help of the ASTRA code [7]. This code allows solving the set of equations of heat flux balance in the electron and ion channels and the poloidal magnetic field diffusion together with the Grad-Shafranov equilibrium equation. Both direct and inverse transport problems can be solved with the ASTRA code. The solution of the direct problem implies the determination of spatial distributions of the plasma parameters and their temporal variation on the basis of given spatial distributions of transport coefficients. The aim of the inverse problem is to determine the transport coefficients based on the spatial distributions of the plasma parameters (temperature, concentration, current density) measured in the experiment. For the discharge with observed TAE, the inverse transport problem was solved as the first step. Then the experimental profiles of electron temperature and density were recalculated as functions of a minor radius, taking into account the Shafranov shift, and, finally, as functions of a flux coordinate as the solution of the direct transport problem.

Fig. 1 shows the experimental profiles of electron temperature and density at different times that were used for modeling. The direct transport problem was solved for ions under the assumption of the neoclassical beha-

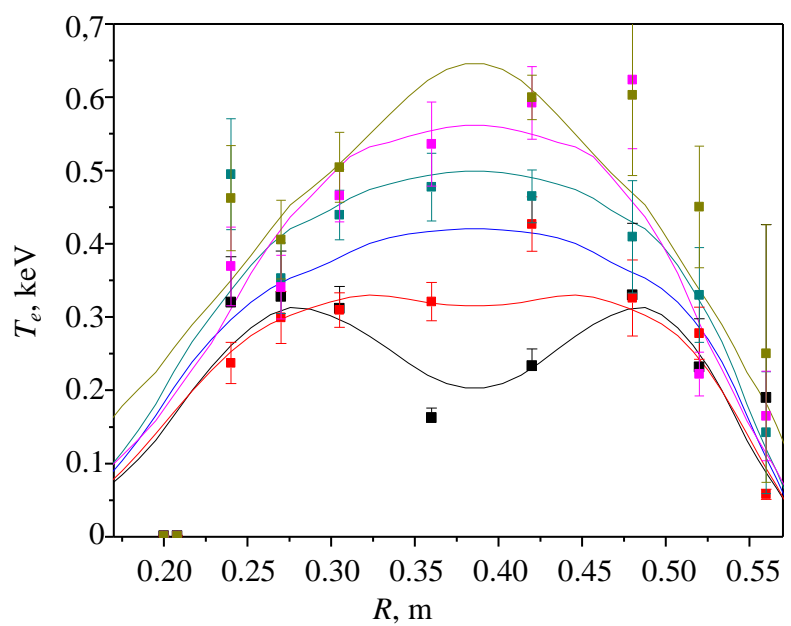

$a$

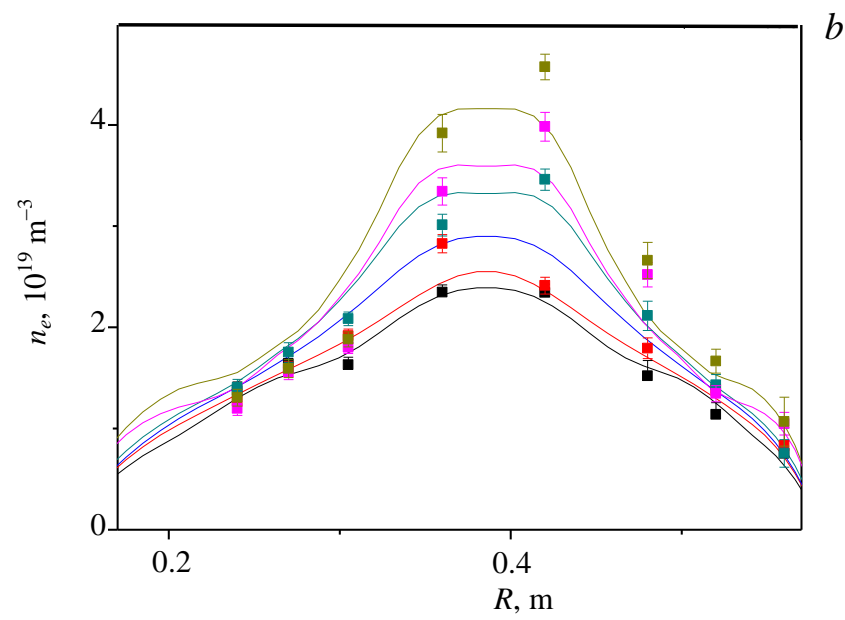

Fig. 1. Experimental profiles of electron temperature $(T)(a)$ and density $(n)(b)$ as functions of the major radius in the equatorial plane of the

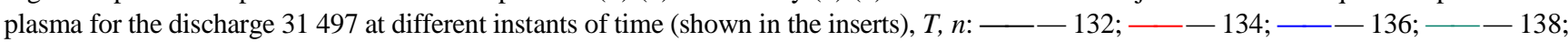
$-140 ;-142$ 
vior of ions on the Globus-M tokamak [8]. For the poloidal magnetic flux diffusion, the direct transport problem was also solved for neoclassical plasma conductivity using the NCLASS code [9]. The ion concentration was calculated from the condition of the plasma quasineutrality. The main impurity was carbon. The distribution of the safety factor along the radius was obtained from the solution of the diffusion equation for the poloidal magnetic flux. The position of the last closed magnetic surface was set based on the EFIT code data [10]. When calculating the absorbed beam power, its component composition and corresponding losses of each of the energy components of the beam were taken into account. The values of the absorbed power were corrected in accordance with the simulation by the full orbit code [11]. The calculated value of the loop voltage of the plasma column with the internal inductance correction was compared with the experimental one, which made it possible to determine the average value of the effective charge of the plasma. As a result, the electron temperature and density distributions in the flux coordinate were obtained, shown in Fig. 2. At the time close to the moment of TAE development the safety factor profile was calculated both in minor radius and in flux coordinate. These profiles along with the data from the EFIT code formed the basis for calculating the Alfvén continuum and the structure of TAE.
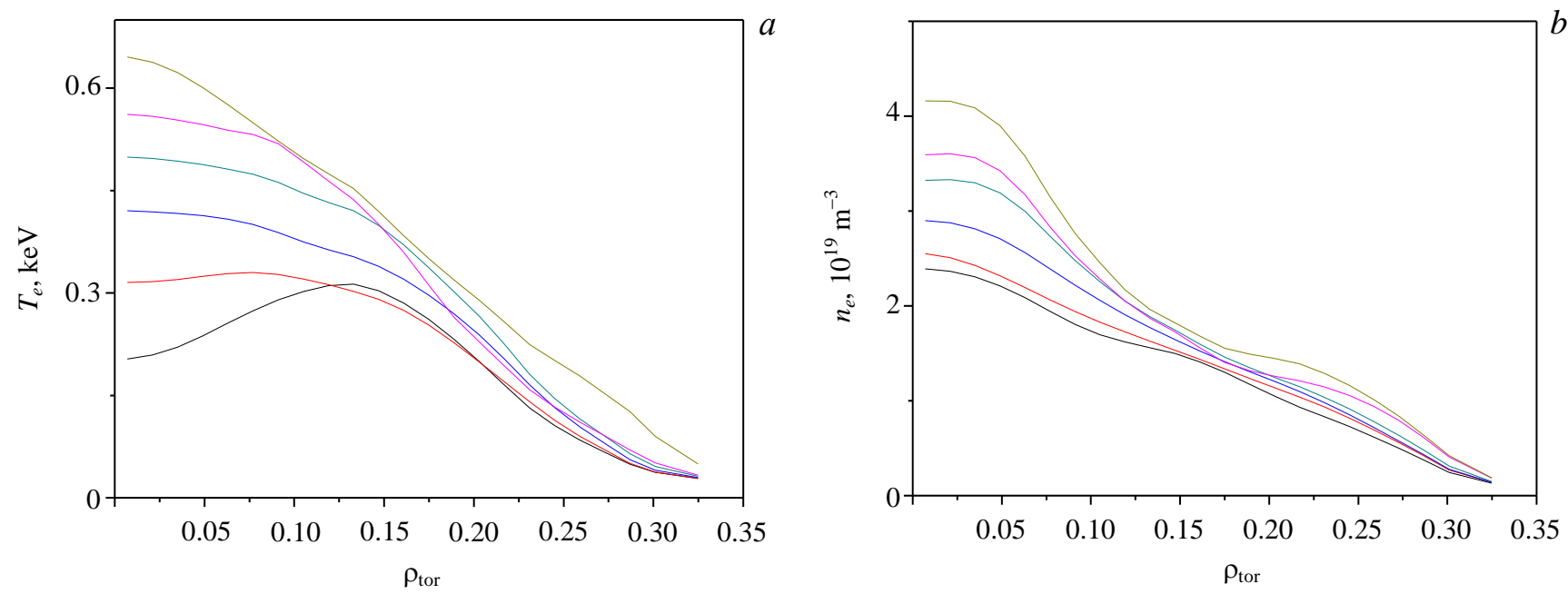

Fig. 2. Experimental profiles of electron temperature $(a)$ and density $(b)$ as functions of averaged minor radius $\rho_{\text {tor }}=\sqrt{\Phi /\left(\pi B_{0}\right)}$, where $\Phi$ is the toroidal flux of the magnetic field inside the magnetic surface, $B_{0}$ is the vacuum toroidal magnetic field in the center of the plasma for the discharge 31497 at different instants of time (shown in the inserts), $T, n$ : 132 ; 134 136 ; $-138$ $-140$ 142. The Shafranov shift is taken into account

\section{ADAPTATION OF THE CODE KINX FOR CALCULATING TAE WITH THE GLOBUS-M PARAMETERS}

The computer code KINX was adapted to calculate the Alfvén continuum and the structures of TAE in a spherical tokamak with Globus-M parameters. Based on the modified KINX stability code and the CAXE code for calculating equilibrium configurations on grids adapted to magnetic surfaces, and Matlab functions for calculating the continuous spectrum and visualization of the results, a software package was prepared for analyzing the structure of the MHD spectrum in spherical tokamaks. As input data for the calculation of equilibrium configurations, the CAXE code uses output files (EQDSK) of the equilibrium reconstruction code EFIT, from which the coordinates of the plasma boundary coinciding with the separatrix of the poloidal magnetic field and the reconstructed profile of the toroidal current density are used. Additional experimental data provide information on the electron temperature and density profiles.

The CAXE code for given boundary coordinates and flux functions of the pressure gradient and plasma poloidal current taken from the EQDSK files provides a highly accurate calculation of the plasma equilibrium required for the subsequent calculation of the continuous and full MHD spectra. Fig. 3 shows the plasma profiles and 
the level lines of the local shear of the magnetic field in such an equilibrium. Herein the mass density profile is restored by mapping and smoothing the experimental data to magnetic surfaces. The approximation of the electron temperature and density by means of functions associated with magnetic surfaces is shown in Fig. 2.
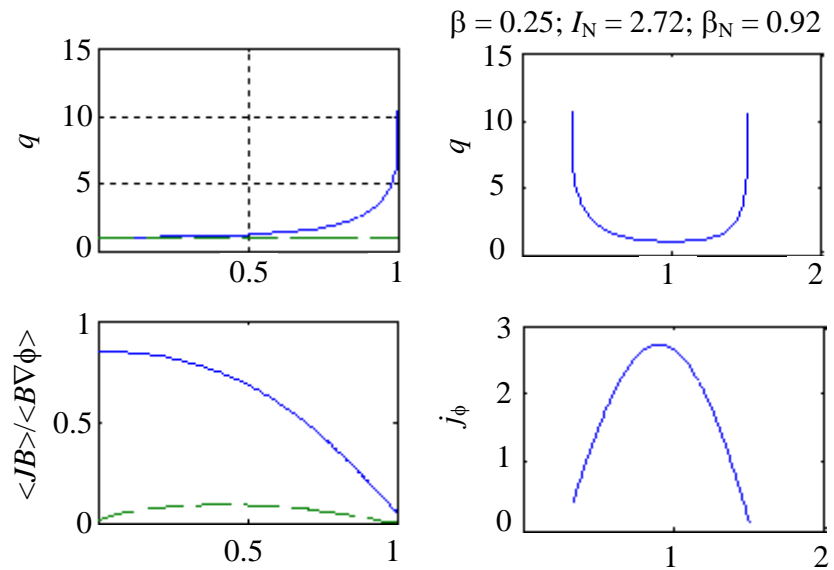

$a$
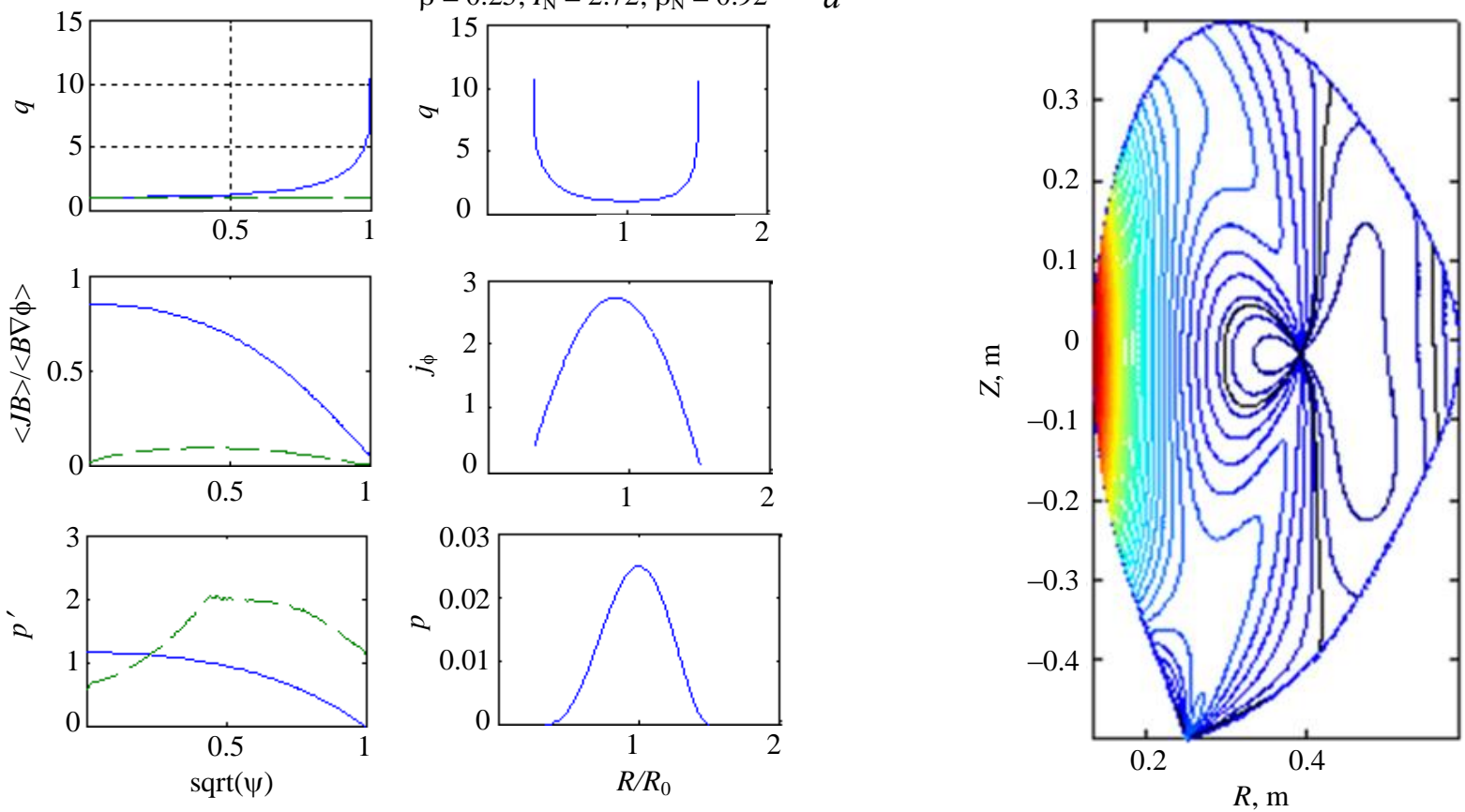

Fig. 3. The plasma profiles as a functions of the normalized poloidal flux $(a)$ and the level lines of the local shear of magnetic field lines in the toroidal cross-section $(b)$ for the reconstructed equilibrium from the discharge of 31497 at $142 \mathrm{~ms}$

The problem of calculating the continuous spectrum reduces to solving a series of one-dimensional spectral problems on each magnetic surface for the resulting equilibrium configurations [5]:

$$
\begin{aligned}
& \omega^{2} \rho \frac{|\nabla \psi|^{2}}{B^{2}} \xi+(\mathbf{B} \nabla)\left(\frac{|\nabla \psi|^{2}}{B^{2}} \mathbf{B} \nabla\right) \xi+\Gamma p \kappa(\nabla \cdot \xi)=0 ; \\
& \omega^{2} \rho \frac{\kappa}{\Gamma p} \xi+\omega^{2} \rho \frac{\Gamma p+B^{2}}{\Gamma p B^{2}}(\nabla \cdot \xi)+(\mathbf{B} \nabla)\left(\frac{1}{B^{2}} \mathbf{B} \nabla\right)(\nabla \cdot \boldsymbol{\xi})=0,
\end{aligned}
$$

where the time dependence $\exp (i \omega t)$ is assumed for the plasma displacement vector from the equilibrium position, $\xi, \xi=\frac{\mathbf{B} \times \nabla \psi}{|\nabla \psi|^{2}} \xi ; \mathbf{B}-$ the equilibrium magnetic field; $\psi-$ the poloidal flux function; $p$ - the pressure; $\rho$ - the mass density; $\Gamma$ - the specific heat ratio; $\kappa=2 \frac{\mathbf{B} \times \nabla \psi}{B^{2}}\left(\frac{\mathbf{B}}{B} \nabla\right)\left(\frac{\mathbf{B}}{B}\right)$. In the slow sound approximation [12], $\Gamma p=\omega^{2} \rho$, it follows from (1) that $\nabla \cdot \xi=-\frac{B^{2}}{\Gamma p+B^{2}} \kappa \xi$, and

$$
\omega^{2} \rho \frac{|\nabla \psi|^{2}}{B^{2}} \xi+(\mathbf{B} \nabla)\left(\frac{|\nabla \psi|^{2}}{B^{2}} \mathbf{B} \nabla\right) \xi-\frac{\Gamma p B^{2} \kappa^{2}}{\Gamma p+B^{2}} \xi=0,
$$

which determines the Alfvén spectrum in this approximation. At the next step with the help of Matlab packages for solving spectral problems, a prescribed number of eigenvalues of the one-dimensional operator are calculated on each magnetic surface, which determine the branches of the continuous spectrum both in the incompressible plasma approximation (Alfvén continuum) and with compressibility (hybrid Alfvén/sound continuum). In the latter case, in addition to the mass density profile, experimental information on the temperature of the plasma is used to reconstruct the pressure profile. We note that the assumption of an ion temperature equal to half 
the electron temperature in deuterium plasma gives a good coincidence of the pressure profiles obtained from experimental measurements and reconstructed profiles based on EFIT data, see Fig. 4.

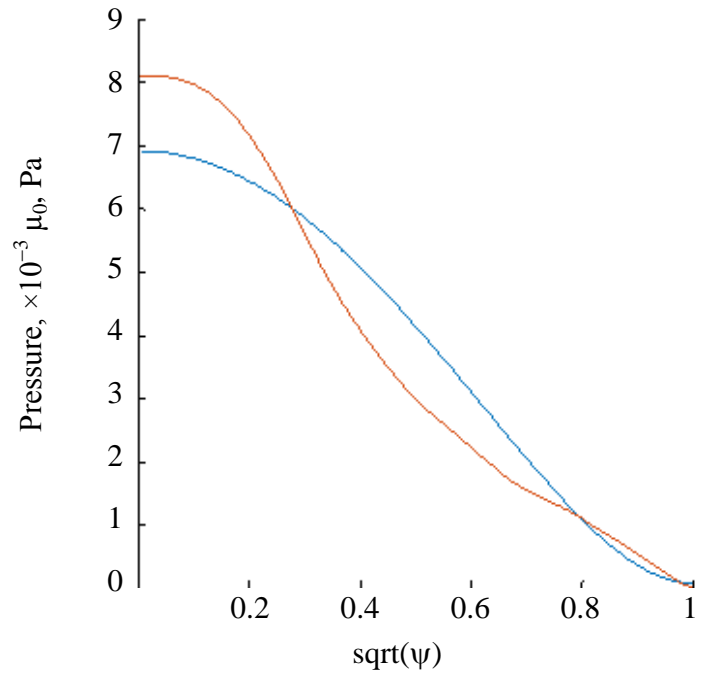

$a$

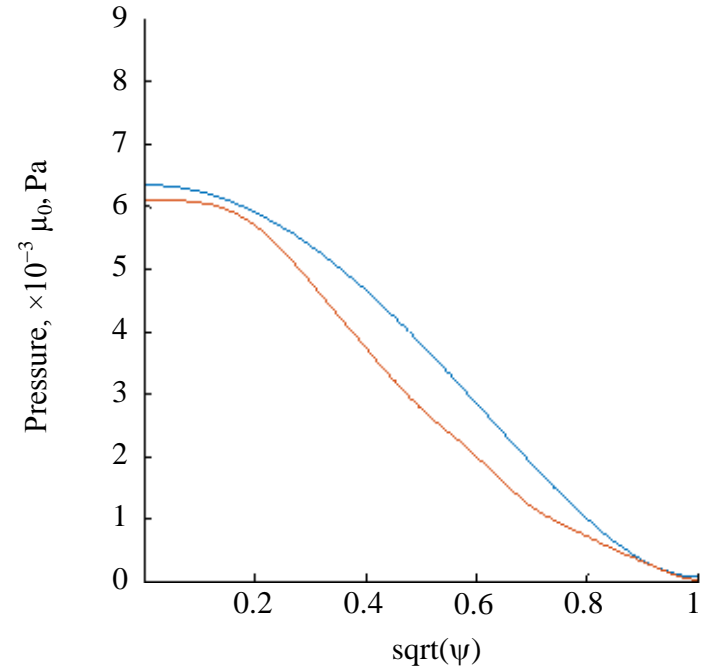

Fig. 4. Comparison of pressure profiles as a function of the square root of the normalized poloidal flux for the discharge 31497 at time instants of $142 \mathrm{~ms}(a)$ and $140 \mathrm{~ms}(b)$, assuming the ion temperature equal to half of the electron temperature: -- EFIT; $-T_{i} / T_{e}=0.5$

The next step in the analysis of the structure of the MHD spectrum is direct calculations using the twodimensional stability code KINX [13]. The KINX code was modified to use the experimental pressure profile in the term $\Gamma p_{\text {exp }}|\nabla \cdot \xi|^{2}$ for a compressible plasma displacement $\xi$ in the perturbed potential energy functional:

$$
\delta W_{p}=\frac{1}{2} \int_{V_{p}}\left\{|\nabla \times(\xi \times \mathbf{B})|^{2}-(\nabla \times \mathbf{B}) \nabla \times(\xi \times \mathbf{B}) \times \xi+\xi \nabla p(\nabla \cdot \xi)+\Gamma p_{\exp }|\nabla \cdot \xi|^{2}\right\} d V .
$$

Extensive calculations of the eigenvalues of a two-dimensional MHD operator are carried out in the range of TAE gaps in the continuous spectrum. In this case, the data on the calculated eigenfunctions with frequencies from a given interval are stored, and the localization of global modes can be analyzed using the Matlab graphical packages. The sensitivity of the TAE frequencies to changes in the boundary conditions is studied by specifying the position of an ideally conducting wall in a vacuum around the plasma column, starting from the wall at the plasma boundary (fixed boundary) to a wall similar to the plasma boundary at a finite distance from it. To calculate the equilibrium and stability, a separatrix of the magnetic field with an $\mathrm{X}$ point on the plasma boundary is chosen as the boundary of the divertor plasma. In the case of free boundary, the sensitivity to the choice of the last closed magnetic surface is verified by selecting the boundary as the magnetic surface with the specified fraction of the poloidal magnetic flux inside the separatrix.

\section{RESULTS OF CALCULATIONS IN COMPARISON WITH EXPERIMENTAL DATA}

To determine the conditions for the excitation of TAEs and their influence on the confinement of fast particles in the spherical Globus-M tokamak [1], the MHD spectra of the reconstructed divertor equilibrium configurations with the safety factor on the magnetic axis $q_{0}>1$ were calculated using the adapted version of the code KINX. Due to axial symmetry of the considered equilibria the spectrum splits into independent toroidal har-mo-nics with wave numbers $n$. The sensitivity of the frequencies of TAE with a toroidal wave number $n=1$ to the type of boundary conditions and the choice of the boundary surface was studied. It is found that the frequencies of the modes with dominant poloidal harmonics $m=1$ and $m=2$ in the continuum gap are much higher than those observed in the spectrograms of the signals from Mirnov coils, especially under the assumption of a free plasma boundary and taking into account its compressibility. TAE with lower frequencies and with higher poloidal wave numbers localized near the plasma boundary can be responsible for the oscillations observed in the experiment. However, for these modes a coupling to the continuum and, possibly, the associated damping are expected. 
In experiments with the neutral beam injection, instabilities excited by fast ions in the frequency range $50-200 \mathrm{kHz}$, which were identified as toroidal Alfvén eigenmodes [1,2], were observed in the early stage of the Globus-M tokamak discharge. For the simulation, a discharge was selected with the injection of a hydrogen beam into a deuterium plasma (see Fig. 2, e from [1], shot 31 497), in which the main intense mode develops at a frequency of $\sim 100 \mathrm{kHz}$. Small aspect ratio of the plasma in a spherical tokamak leads to the appearance of a wide gap in the continuum due to the toroidal coupling of the modes with neigh boring numbers of the poloidal harmonics $m$ and $m+1$. Fig. 5 shows the structure of the continuous Alfvén spectrum (the specific heat ratio $\Gamma=0$ ) calculated by the KINX code using the experimental mass density profile for a quasineutral deuterium plasma. In this gap, there are several global TAE near the upper and lower boundaries of the continuum (the black horizontal lines in Fig. 5). We note that the boundary conditions at

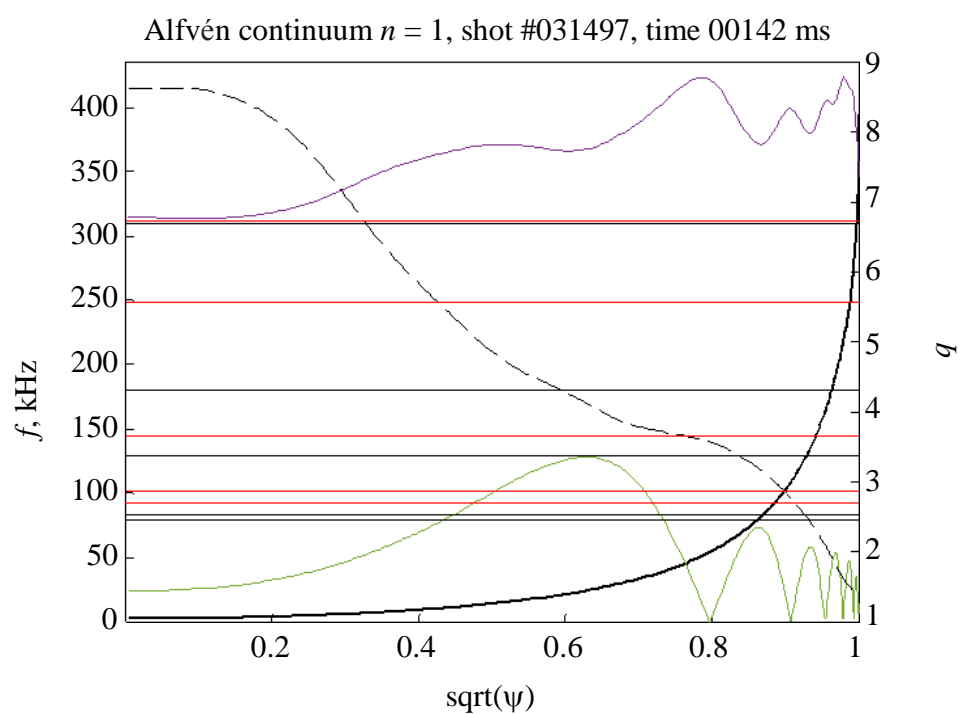

Fig. 5. The structure of the continuous Alfvén spectrum for the toroidal wave number $n=1$. The profile of the safety factor $q$ is shown by a thick line, the experimental mass density profile normalized to the Alfvén frequency in the center of the plasma is the dashed line. The horizontal lines correspond to the frequencies of the global modes

Toroidal mode number $n=1$

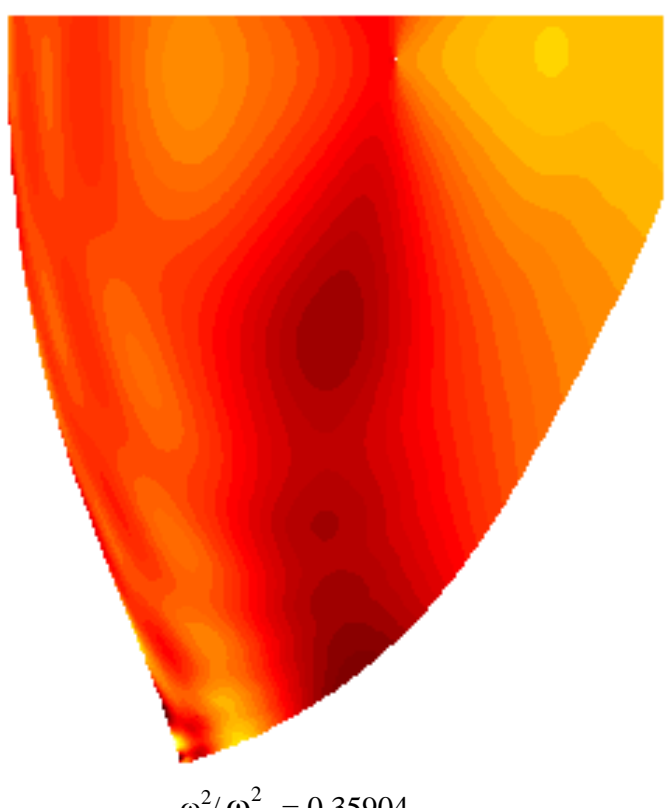
the free boundary of the plasma (the radius of the conducting wall $1.3 a$ ) lead to a noticeable increase in the frequencies of the global modes (Fig. 5, red horizontal lines).

At the same time, choosing a separatrix of the magnetic field or a magnetic surface inside the separatrix, determined by the fraction of the poloidal flux, as a plasma boundary does not lead to a significant change in the frequencies of the external modes, which is a consequence of the high shear near the plasma boundary of the spherical tokamak. A comparison of the structure of the displacements for different choices of the last closed flux surface is shown in Fig. 6 for the TAE which experiences the strongest increase of the frequency with the free boundary of plasma as compared to the fixed boundary.

Toroidal mode number $n=1$

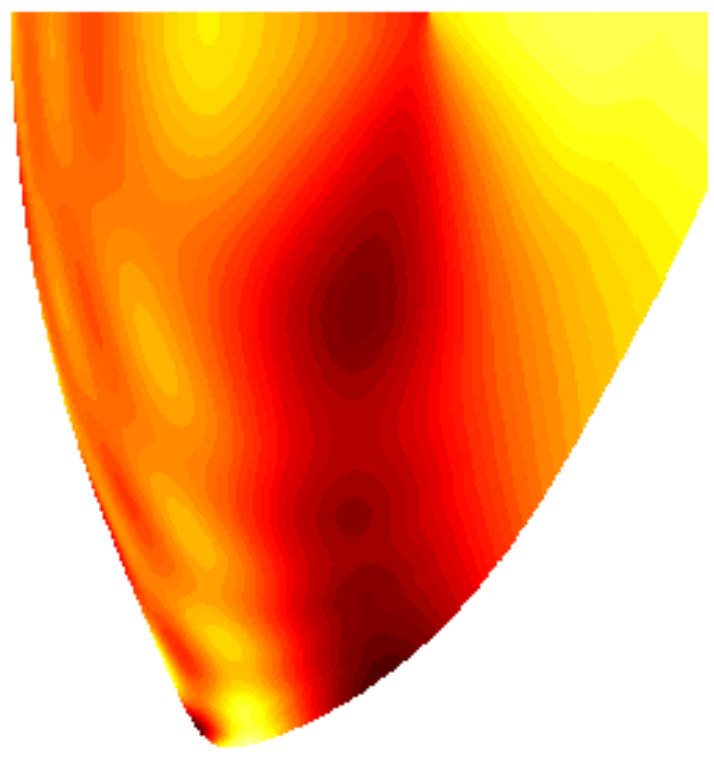

$\omega^{2} / \omega_{\mathrm{A}}^{2}=0.36262$

Fig. 6. Comparison of the plasma displacements for a different choice of the plasma boundary. The level lines of the displacement normal to magnetic surfaces are shown: the separatrix at the plasma boundary, the eigenfrequency is $248 \mathrm{kHz}(a)$, and the boundary is determined by the fraction of the poloidal flux inside the separatrix, the eigenfrequency is $249 \mathrm{kHz}(b)$ 
The structures of the modes with the boundary conditions corresponding to the fixed and free boundary of the plasma are shown in Fig. 7. In addition to TAE with frequencies falling into the main TAE gap located near
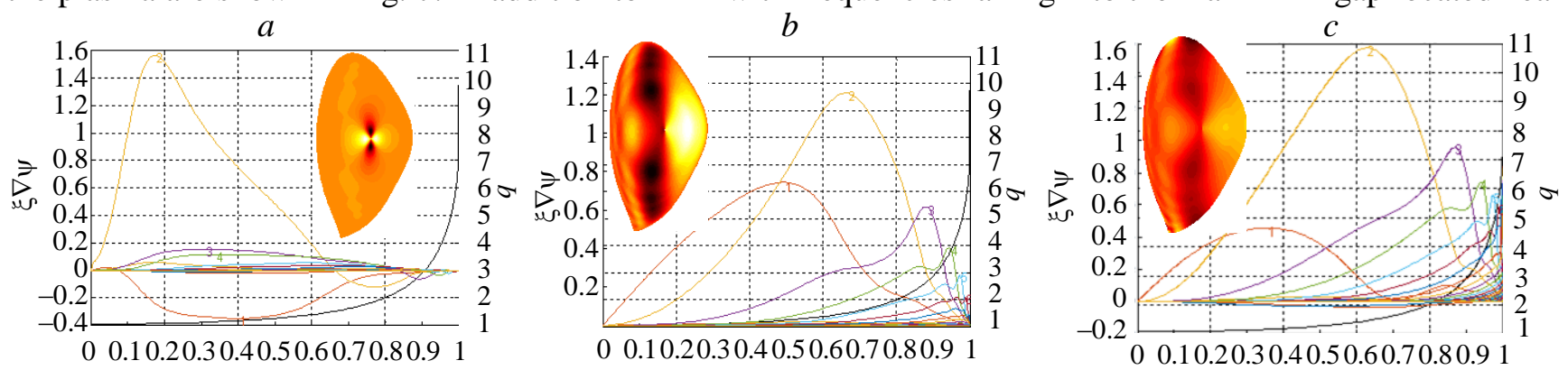

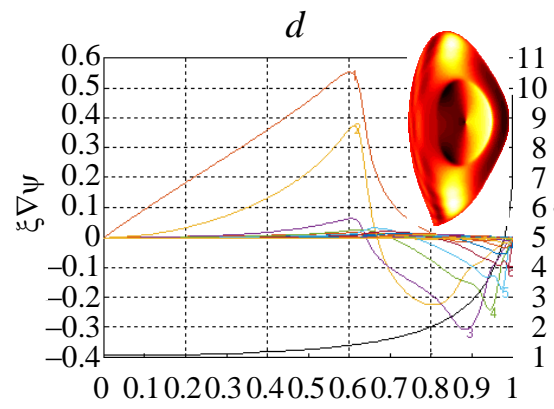

$g$

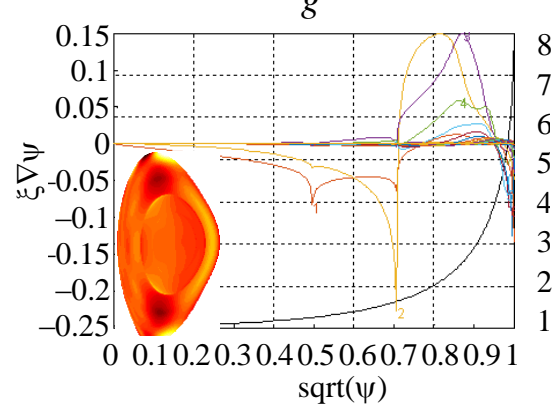

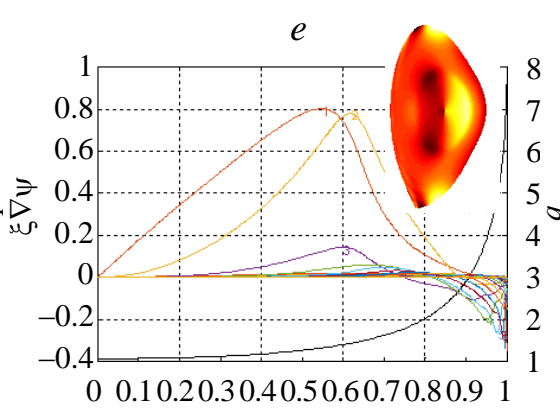

$h$

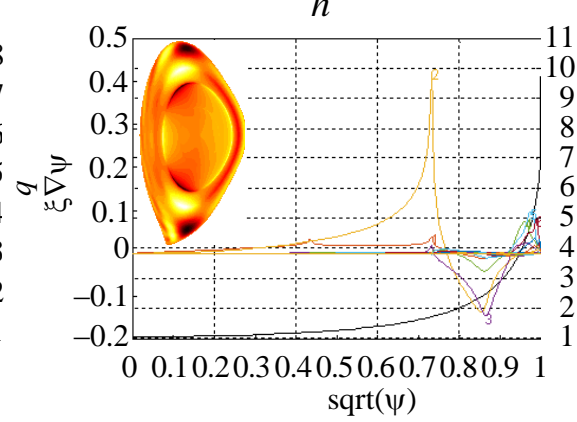

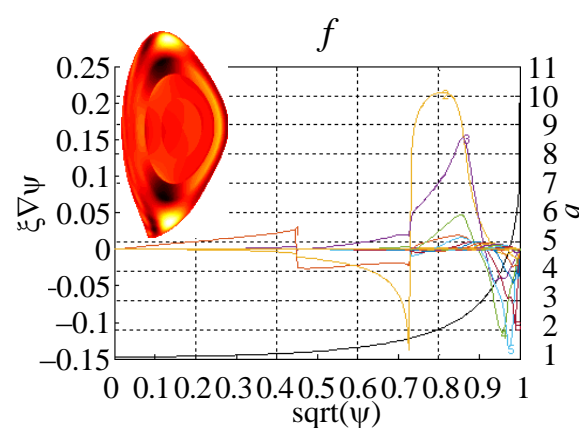

Fig. 7. The structures of the TAE for the plasma in discharge 31497 (time $142 \mathrm{~ms}$ ) with the boundary conditions corresponding to the fixed and free boundary of the plasma and the following frequencies: $391 \mathrm{kHz}(a), 181 \mathrm{kHz}(b)$, $-248 \mathrm{kHz}(c), 129 \mathrm{kHz}(d)$, fixed boundary, $144 \mathrm{kHz}(e)$, free boundary, $83 \mathrm{kHz}(f)$, fixed boundary, $102 \mathrm{kHz}(g)$, free boundary, $79 \mathrm{kHz}(h)$, fixed boundary. Harmonics of the plasma displacement normal to the magnetic surfaces $\xi \nabla \psi$ in straight field line coordinates and the level lines of $\xi \nabla \psi /|\nabla \psi|$ are shown

the magnetic surface with $q=1.5$, in the ideal MHD spectrum there are global modes with frequencies falling into the continuous spectrum. Figs. 7, $f, g, h$ show the structures of such modes with large numbers of poloidal harmonics, which correspond to the toroidal «reconnection» of the continuum branches at the magnetic surfa-ces with $q=2.5,3.5$. The flux grid with 256 radial (packed to the boundary) by 256 poloidal intervals has been checked to be quite sufficient for an accurate resolution of the eigenfunctions in the TAE gap. For the modes with the frequencies in the continuum no attempt was made either to resolve the coupling to singular continuum modes or to estimate the corresponding continuum damping in the simplistic ideal MHD model. The purpose of the presented spectrum calculations is just a demonstration of the existence of the higher-m TAE inside the continuum and its frequency dependence on the boundary conditions (compare Fig. 7, $f$ and Fig. 7, $g$ ).

The assumption of plasma compressibility (the specific heat ratio $\Gamma=5 / 3$, the pressure profile obtained from the data on the electron temperature, assuming $T_{i} / T_{e}=0.5$ ) leads to the Alfvén and sound continua coupling (Fig. 8). In addition to the branches

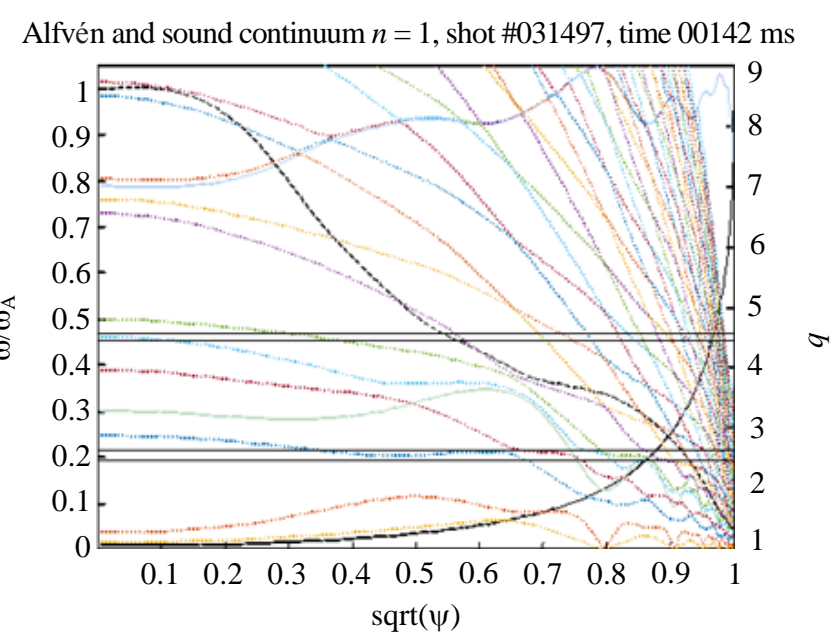

Fig. 8. The structure of the continuous Alfvén/sound spectrum for the toroidal wave number $n=1$ for the compressible plasma (discharge 31497 , time $142 \mathrm{~ms}$, Alfvén frequency at the center of the plasma $\omega_{\mathrm{A}}=414 \mathrm{kHz}$ ). The profile of the safety factor $q$ is shown by the thick line, the experimental mass density profile normalized to its value in the center of the plasma is dashed line. The branches of the continuum are shown in multicolored dots, the solid lines correspond to the branches of the Alfvén continuum in the limit of slow sound. Horizontal lines correspond to the frequencies of global modes 
of the Alfvén/sound continuum, the frequencies of the Alfvén branch in the limit of slow sound are also shown. Analogs of the incompressible global modes with frequencies close to experimental ones were found for the compressible plasma with finite pressure (compare Fig. 7, $h$ and Fig. 9, $b$ ). Figs. 9, 10 show the structures of such modes with frequencies and localization corresponding to the local maxima of the branches of the Alfvén/sound continuum for two instants of time. In this case, the mode with a lower frequency and a large poloidal harmonic $m=1$ is apparently the result of the coupling of Alfvén and sound modes, as in the case of BAAE (Beta-induced Alfvén Acoustic Eigenmode) modes [14] with lower frequencies.
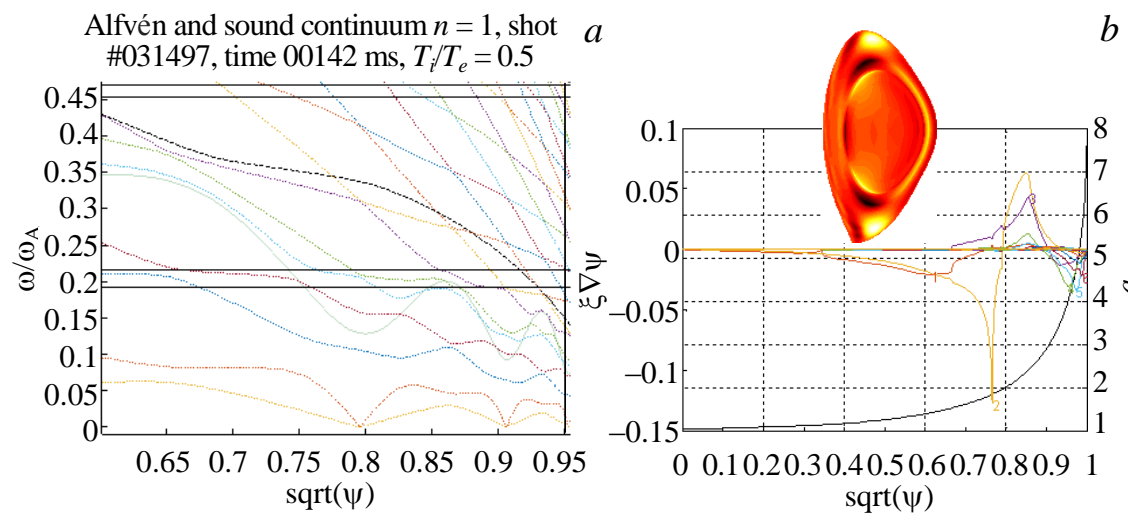

$b$

$c$

Fig. 9. An enlarged fragment of the structure of the continuous Alfvén/sound spectrum for the toroidal wave number $n=1$ for the compressible plasma (discharge 31 497, time $142 \mathrm{~ms}$, Alfvén frequency at the center of the plasma $\left.\omega_{\mathrm{A}}=414 \mathrm{kHz}\right)(a)$ and the structure of global modes with low frequencies $89 \mathrm{kHz}(b), 80 \mathrm{kHz}(c)$

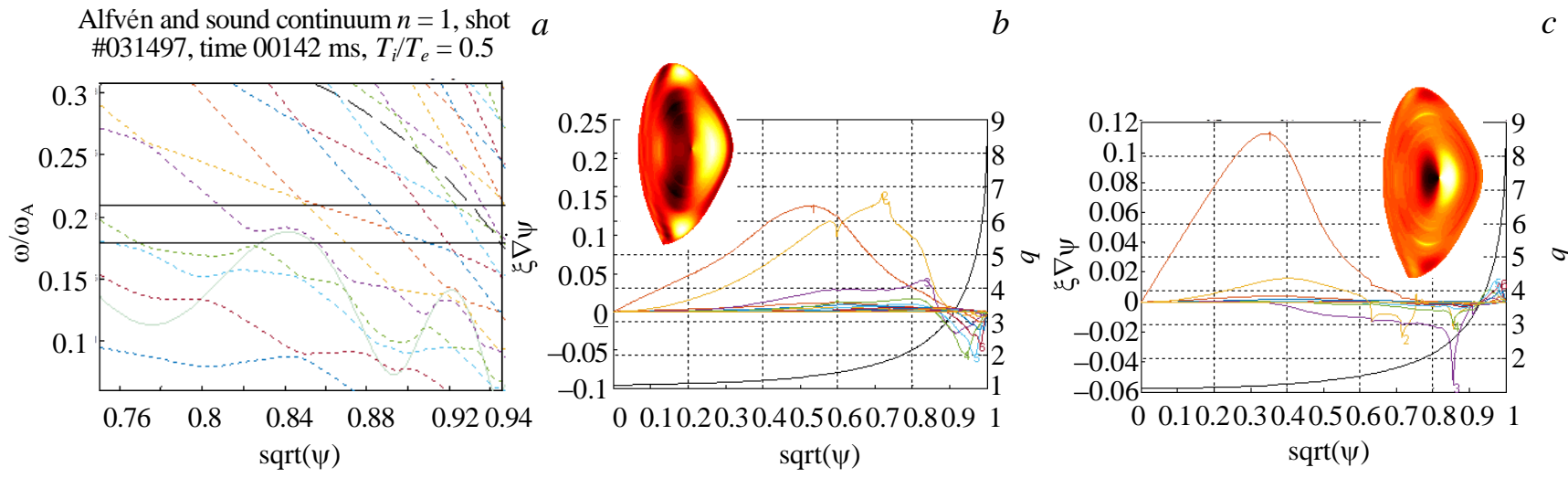

Fig. 10. An enlarged fragment of the structure of the continuous Alfvén /sound spectrum for the toroidal wave number $n=1$ for the compressible plasma (discharge 31497 , time $140 \mathrm{~ms}$, Alfvén frequency at the center of the plasma $\left.\omega_{\mathrm{A}}=450 \mathrm{kHz}\right)(a)$ and the structure of global modes with low frequencies: $94 \mathrm{kHz}(b), 81 \mathrm{kHz}(c)$

The results of the calculations allow to conclude that the oscillation frequencies observed in the experiment are rather close to the frequencies of the TAE localized near the plasma boundary in the region of magnetic surfaces with the safety factor values $q=2.5,3.5$. The frequencies of these modes fall into a continuous spectrum, which can lead to their enhanced damping. However, as discussed in [15], the Landau damping coefficients on thermal ions can be low due to a change in the mode polarization during the interaction of a wave with energetic particles.

\section{CONCLUSIONS}

The theoretical analysis of the experiments on TAE excitation by means of NBI in the Globus-M spherical tokamak was carried out. The cases with mainly single-mode instabilities with toroidal number $n=1$ were investigated in accordance with the linear theory predictions for the Globus-M conditions. The experiments on Globus-M have shown that essential losses in the neutron rate (up to 25\%) associated with TAE are possible due to the single-mode TAE development even in the absence of the Alfvén avalanches. The MHD spectrum analysis demonstrated that the TAE localized near the plasma boundary in the region of magnetic surfaces with the safety factor values $q=2.5,3.5$ can be responsible for the fast particle losses. The open question is the influence of TAE on fast particle losses in future CNS designs. One cannot exclude an essential drop of the neutron rate 
due to the TAE development. Experiments on the upgraded tokamak Globus-M2 should provide additional data for extrapolation to the CNS parameters.

This research was supported by the Russian Science Foundation (Grant No. 17-12-01177).

\section{REFERENCES}

1. Petrov Yu.V., Patrov M.I., Gusev V.K. et al. - Fizika plazmy (Plasma Physics), 2011, vol. 37, № 12, pp. 1075-1080 (in Russian).

2. Petrov Yu.V. et al. — J. Plasma Phys., 2015, vol. 81, p. 515810601.

3. Petrov Yu.V., Bakharev N.N., Gusev V.K. et al. — PZhTF (LJTPh), 2014, vol. 40, issue 24, pp. 99-106 (in Russian).

4. Gusev V.K., Martynov A.A., Medvedev C.Yu. et al. — PZhTF (LJTPh), 2018, vol. 44, issue 2, pp. 65-71 (in Russian).

5. Cheng C.Z., Chance M.S. - Phys. Fluids, 1986, vol. 11, p. 3695.

6. Fredrickson E.D. et al. - Nucl. Fusion, 2013, vol. 53, p. 013006.

7. Pereverzev G.V., Yushmanov P.N. - Max-Plank IPP Report 5/98, 2002.

8. Avdeeva G.F. et al. - J. Phys. Conf. Ser., 2016, vol. 666, p. 012002.

9. Houlberg W.A. et al. - Phys. Plasmas, 1997, vol. 4, p. 3230.

10.Lao L.L. et al. - Nucl. Fusion, 1985, vol. 25, p. 1611.

11.Bakharev N.N. et al. — Nucl. Fusion, 2015, vol. 55, p. 043023.

12.Chu M.S., Greene J.M., Lao L.L., Turnbull A.D., Chance M.S. - Physics of Fluids B, 1992, vol. 4, p.3713.

13.Degtyarev L., Martynov A., Medvedev S., Troyon F., Villard L., Gruber R. - Comput. Phys. Comm., 1997, vol. 103, pp. 10-27.

14.Gorelenkov N.N. et al. - Phys. Lett. A, 2007, vol. 370/1, p. 70.

15.Liu Y., Lin Z., Zhang H., Zhang W. — Nucl. Fusion, 2017, vol. 57, p. 114001.

\section{AUTHORS}

Medvedev S.Yu. M.V. Keldysh Institute of Applied Mathematics RAS, Miusskaya sq. 4, 125047 Moscow, Russia; NRC "Kurchatov Institute", pl. Akademika Kurchatova 1, 123182 Moscow, Russia; medvedev@a5.kiam.ru

Martynov A.A. M.V. Keldysh Institute of Applied Mathematics RAS, Miusskaya sq. 4, 125047 Moscow, Russia; NRC “Kurchatov Institute”, pl. Akademika Kurchatova 1, 123182 Moscow, Russia; martynov@a5.kiam.ru

Gusev V.K. A.F. Ioffe Physical-Technical Institute of the Russian Academy of Sciences, Politekhnicheskaya ul. 26, 194021 St. Petersburg, Russia

Petrov Yu.V. A.F. Ioffe Physical-Technical Institute of the Russian Academy of Sciences, Politekhnicheskaya ul. 26, 194021 St. Petersburg, Russia

Patrov M.I. A.F. Ioffe Physical-Technical Institute of the Russian Academy of Sciences, Politekhnicheskaya ul. 26,194021 St. Petersburg, Russia; michael.patrov@mail.ioffe.ru

Tel'nova A.Yu. A.F. Ioffe Physical-Technical Institute of the Russian Academy of Sciences,

Politekhnicheskaya ul. 26, 194021 St. Petersburg, Russia

Ivanov A.A. M.V. Keldysh Institute of Applied Mathematics RAS, Miusskaya sq. 4, 125047 Moscow, Russia; aai@a5.kiam.ru

Poshekhonov Yu.Yu. M.V. Keldysh Institute of Applied Mathematics RAS, Miusskaya sq. 4, 125047 Moscow, Russia; naida@a5.kiam.ru 
Received 18 March 2018

Problems of Atomic Science and Technology Ser. Thermonuclear Fusion, 2018, vol. 41, issue 2, pp. 95-104 